\title{
INDICADORES DE SOSTENIBILIDAD PARA EL ECOTURISMO EN MÉXICO: ESTADO ACTUAL
}

\author{
Sustainability Indicators for Ecotourism in Mexico: Current State \\ Edali Camacho-Ruiz \\ Arturo Carrillo-Reyes \\ Tamara Mila Rioja-Paradela \\ Eduardo E. Espinoza-Medinilla
}

\begin{abstract}
Resumen: El manejo actual de los centros ecoturísticos se basa en el conocimiento intrínseco de los espacios naturales y de los recursos culturales y económicos de las comunidades locales donde se encuentran. No obstante, el conocimiento y uso de indicadores que permitan el diagnóstico y evaluación de la sostenibilidad aún están limitados. Se hace una revisión sobre el uso de indicadores turísticos de sostenibilidad en México y se analizan los vacíos de información, además de examinar algunas implicaciones para el ecoturismo. Se propone que la construcción de un sistema de indicadores con generalidades y especificidades promovería el manejo más sostenible del turismo y podría representar un medio de diálogo entre los actores involucrados.
\end{abstract}

Palabras clave: ecoturismo, desarrollo sostenible, indicadores ambientales, política ambiental.

Abstract: Current management of ecotourism centers is based on intrinsic knowledge of natural and economic areas, cultural resources of local communities where tourism is developed. However, knowledge about the sustainability of these centers is far from complete, and the existence and use of indicators for diagnosis and evaluation to determine the distance to meet sustainability goals. This manuscript is a review of the use of tourism sustainability indicators in México and it attempts to analyze the information gaps; it also suggests implications for ecoturism activity for ecotourism activity. The article proposes that the construction of a system of indicators with generalities and specificities not only promotes the sustainable management of the destination, but also can be a means to dialogue among social actors involved.

Keywords: ecotourism, sustainable development, environmental indicators, environmental policy.

Edali Camacho Ruiz, estudiante de la Maestría en Ciencias en Desarrollo Sustentable de la Universidad de Ciencias y Artes de Chiapas, México. Tema de especialización: sostenibilidad, ecoturismo. Correo electrónico: edali.cr@gmail.com.

Arturo Carrillo Reyes, doctor en Ciencias en Ecología y Desarrollo Sustentable por El Colegio de la Frontera Sur, México. Profesorinvestigador en el Posgrado en Ciencias en Desarrollo Sustentable de la Universidad de Ciencias y Artes de Chiapas, México. Temas de especialización: conservación y manejo de vida silvestre, desarrollo sustentable. Correo electrónico: arturo.carrillo@unicach.mx.

Tamara Mila Rioja Paradela, doctora en Ciencias en Ecología y Desarrollo Sustentable por El Colegio de la Frontera Sur, México. Profesora en el Posgrado en Ciencias en Desarrollo Sustentable de la Universidad de Ciencias y Artes de Chiapas, México. Temas de especialización: manejo y conservación de flora y fauna, desarrollo sustentable. Correo electrónico: tamara.rioja@unicach.mx.

Eduardo E. Espinoza Medinilla, doctor en Ciencias Biológicas por la Universidad Autónoma de Querétaro, México. Profesor en el Posgrado en Ciencias en Desarrollo Sustentable de la Universidad de Ciencias y Artes de Chiapas, México. Temas de especialización: biología de la conservación. Correo electrónico: eduardo.espinoza@unicach.mx.

Enviado a dictamen: 2 de junio de 2015. Aprobación: 30 de septiembre de 2015. Revisiones: 1 . 


\section{Introducción}

L a biodiversidad ecosistémica en México ha permitido el desarrollo de la actividad ecoturística hacia un nuevo modelo de turismo sostenible (Blancas et al., 2010: 85). En Chiapas esto cobra mayor relevancia ya que el estado posee un alto potencial turístico capaz de generar condiciones de bienestar para las comunidades locales (UNWTO, 2009: 22). El arraigo al nuevo modelo permite mantener los espacios naturales y socioculturales de los destinos y ver más allá de una actividad basada en el paradigma de una sostenibilidad débil (Constanza, 1991: 336; Jiménez, 2000: 294; Luffiego et al., 2000: 480). Es en este contexto en el que surgen los indicadores de sostenibilidad turística, como herramientas de evaluación que permiten cuantificar los impactos de dicha actividad, además de encauzar y reorientar estrategias y actividades para asegurar la experiencia satisfactoria de los visitantes y para conservar y proteger los recursos naturales, así como para obtener propuestas de mejora en la calidad de vida de las comunidades locales y los gestores de la actividad a través de los beneficios económicos logrados (SECTUR, 2014).

No obstante, a pesar de su relevancia actualmente no existe una referencia documental que concentre la información disponible respecto al estado de los indicadores disponibles, así como sus fortalezas y debilidades, frente a una actividad compleja y en constante crecimiento. En el presente documento se analiza el estado actual de los indicadores de sostenibilidad turística y las áreas de oportunidad en su praxis, además de proponer una metodología para su diseño en el proceso de planificación de los destinos ecoturísticos desde la perspectiva de la sostenibilidad.

\section{Desarrollo}

La palabra "desarrollo" frecuentemente se asocia al crecimiento económico y sociopolítico (Guillén, 2004: 26), y a la modernización de las ciudades y sociedades (Rostow, 1960: 272). Es el resultado de la adopción del modelo europeo o americano por su concepción de prosperidad económica y estabilidad política. Sin embargo, en las definiciones del concepto se elimina parte de la culturalidad de la sociedad, por lo que disminuye la capacidad de llevar a la práctica un modelo propio de desarrollo basado en los recursos de que disponen las distintas sociedades (Rostow, 1960: 272). Cada región del planeta debe desplegar una apropiación única de acuerdo con sus condiciones actuales independientes (Rostow, 1960: 272), articulándose aspectos de crecimiento socioeconómico, políticoadministrativo y ambiental, tomando en consideración que del estado y condiciones del ambiente dependen las posibilidades de desarrollo social, no sólo en regiones con poblaciones altamente marginadas, sino en todas las sociedades (González et al., 2007: 10). Es decir, para entender el desarrollo, el progreso, el crecimiento económico y la colectividad social hay que tomar en cuenta la sostenibilidad (Angulo, 2010: 4).

\section{Sustentabilidad y sostenibilidad}

Las palabras "sostenible" y "sustentable" se han adaptado del término sustainable development, mediante una traducción deficiente de la palabra sajona al castellano (Wolfernsberger, 2005: 93). En América Latina el término "sustentabilidad" se emplea como sinónimo del de "sostenibilidad". Sin embargo, en este artículo se utilizará el término "sostenible" para referirse a sostener el desarrollo manteniendo su sustento físico y vital. Como señala Jiménez (2000: 293), no se trata sólo de "sostener" de forma duradera los flujos de producción y de insumos, sino de ajustarlos a la capacidad de existencia y regeneración del capital natural.

Ahora bien, en el tenor de la sostenibilidad existen dos modelos o filosofías: la sostenibilidad débil y la fuerte (Toro, 2007). La primera considera que el desarrollo sostenible es una fase en la que el componente ambiental dentro de la sociedad industrial se toma en cuenta siempre y cuando no afecte la búsqueda del máximo beneficio. En esta postura no se distingue el capital natural del social. El capital natural es un concepto utilizado por los economistas para designar la fuente responsable del flujo de los recursos naturales 
de una sociedad como, por ejemplo, el bosque que produce el flujo de madera (Daly, 1992; Luffiego et al., 2000). La "ecoeficiencia", entendida como la "filosofía de los procesos productivos y las pautas de consumo" (Toro, 2007), se ajusta a esta consideración, ya que una reducción en el uso de la materia y de la energía comporta una alta productividad. Además, en la sostenibilidad débil se hace mayor énfasis en el empleo de la tecnología como herramienta para poder alcanzar la ecoeficiencia y para ampliar y retrasar los límites naturales a un crecimiento indefinido (Toro, 2007). Así, la sostenibilidad débil se fundamenta en una visión antropocéntrica que determina la valoración del capital natural en términos económicos, lo cual fomenta la subvaloración de los recursos naturales que proporcionan procesos vitales y ecológicos insustituibles, los cuales las futuras generaciones no podrán heredar, ni podrán participar en el juego de la valoración del capital natural en cuanto a términos de oferta y demanda, por lo que se concibe como un paradigma mecanicista y reduccionista de la sostenibilidad (Luffiego et al., 2000).

Por otrolado, la sostenibilidadfuertees representada por la economía ecológica, concebida como la ideal y utópica que considera una sostenibilidad global a partir de las sostenibilidades locales, las cuales se alcanzan bajo el supuesto de que el desarrollo socioeconómico es dependiente de los ecosistemas (Naredo, 1996), ya que estos últimos pueden funcionar autónomamente, mientras que el primero no podría hacerlo sin los ecosistemas. Debemos mencionar que, para efectos de este análisis, el acercamiento a la sostenibilidad fuerte en su concepción más completa es el que los autores tienen como referencia. Si bien la visión ideal de dicho acercamiento parece limitar una aproximación objetiva, consideramos que al mismo tiempo fija un paradigma que debe considerarse como un camino por seguir. A pesar de la certeza de que difícilmente se logrará alcanzar con absoluta confianza su cumplimiento, sí es posible dirigirse en ese sentido con el propósito de priorizar el hábitat y sus recursos, de los cuales dependemos, por encima de las utilidades que de estos pueden obtenerse.

\section{Turismo sostenible y ecoturismo}

Se considera la sostenibilidad como un estado ideal, utópico e inalcanzable que descalifica las posibilidades reales de su aplicación a la planificación y gestión de los destinos turísticos. Sin embargo, debe concebirse como un proceso de cambio cualitativo producto del arraigo y de la praxis del concepto, orientado o reorientado hacia objetivos alcanzables dentro del sector (Vera, 2001: 3). Su complejidad en la práctica se debe a la dificultad en el entendimiento y arraigo del concepto por parte de la población local, a la imposición de actividades por actores sociales, y al enaltecimiento y priorización económica sobre los demás ejes de la sostenibilidad (Vera, 2001: 4). Para lograr la sostenibilidad en los destinos turísticos se debe inducir la praxis y operatividad del marco institucional y legal, así como los principios de planificación y gestión del destino (Vera, 2001: 4). Deben tomarse en consideración las necesidades y aspiraciones de la población local y de los visitantes hacia la conservación del patrimonio natural y cultural, y hacia la equidad social, mediante la participación social bajo un enfoque objetivo y metodológico. Además, es necesario medir el alcance de los objetivos fijados para conocer desde y hacia dónde se dirige el desarrollo del destino. Para ello se requiere del diseño, selección, implementación y evaluación de indicadores de sostenibilidad turística.

A este respecto, el ecoturismo es el segmento con mayor crecimiento en el mundo y con mayor potencial para integrar las dimensiones de la sostenibilidad. Es una actividad que, en la mejor de sus acepciones, produce un mínimo impacto en el ambiente, y relaciona aspectos de aprendizaje con la conservación, la comprensión y el aprecio por el entorno y por las culturas que se visitan. Por lo general se establece en zonas vírgenes o bien conservadas, o en zonas territoriales en las quela presencia de seres humanos es mínima. El turista se motiva a educarse y sensibilizarse desde el punto de vista ambiental y cultural a través de la experiencia con la naturaleza (Vanegas, 2006: 15). Además, esta actividad genera ingresos para la conservación y beneficios económicos para las comunidades locales (OMT, 1996: 74). 
La planeación y diseño de destinos de esta naturaleza se sustenta en el conocimiento de los recursos naturales y socioculturales de las comunidades locales para establecer límites de uso (Vanegas, 2006: 27). Para ello, el ordenamiento territorial debe definir el uso del espacio ecoturístico como resultado de la aplicación de técnicas de inventarios de recursos naturales, productivos y sociales, así como de infraestructura. En caso de que el destino se encuentre dentro de áreas protegidas, la zonificación permite priorizar actividades, como la investigación o protección de áreas, para garantizar la continuidad de los procesos ecosistémicos (Vanegas, 2006: 28). Finalmente, la operatividad de cada destino ecoturístico debe regirse bajo reglamentos y normas para personas locales y para visitantes que garanticen su aplicabilidad en las diferentes escalas de operación, calificando la prestación de servicios, la administración, la protección, la restauración de los recursos naturales yla conservación de la identidad cultural (Vanegas, 2006:29).

\section{Indicadores de sostenibilidad}

La complejidad del modelo de desarrollo sostenible radica en ubicar instrumentos de medición y evaluación adecuados que permitan conocer cómo se encuentra un sistema dentro del esquema idealista del desarrollo (Ibáñez, 2012: 102) o de la sostenibilidad. La Comisión de las Naciones Unidas sobre el Desarrollo Sostenible creó una iniciativa de medición y evaluación de los avances del actual modelo llamado indicadores de sostenibilidad. De acuerdo con Sánchez, un indicador es una:

[...] expresión que relaciona variables o atributos estadísticos con el objeto de proveer información oportuna, adecuada, confiable y comparable para el análisis y el monitoreo de una determinada problemática. Son instrumentos de continua medición, interpretación, intervención e impacto que debe repetirse de manera iterativa (Sánchez, 2008:5).

Es decir, los indicadores son herramientas que permiten conocer y analizar información específica sobre determinados aspectos y espacios con base en un valor de referencia (Sánchez, 2008: 5). Para ello se identifican o construyen variables con valor explicativo. Su formulación, aplicabilidad y evaluación son muy importantes ya que representan los compromisos de empresas, instituciones, asociaciones de los sectores públicos y privados y de la comunidad local en conjunto (Sánchez, 2008: 6).

\section{Clasificación de los indicadores de sostenibilidad}

La clasificación de los indicadores de sostenibilidad se basa en diferentes criterios (Ibáñez, 2012: 106). A continuación se mencionan los más importantes:

1. Según la dimensión del desarrollo sostenible al que están orientados: estos indicadores se fundamentan en los alcances temáticos del desarrollo sostenible, social, económico, ambiental, y actualmente se incluye el institucional.

2. Según el sentido teórico y la obtención de datos para su elaboración:

2.a. Indicadores objetivos (duros): son cuantitativos y en ellos se emplea información objetiva obtenida a través de los diferentes métodos de captación y procesamiento.

2.b. Indicadores subjetivos (alternos): son cualitativos y en ellos se emplea información obtenida a partir de opiniones y percepciones de la población respecto a una situación que les involucra.

3. Según el modelo en el que estén basados:

3.a. Basadosenel modelo presión-estado-respuesta (PER): modelo que supone que las actividades humanas ejercen presión sobre el ambiente provocando cambios en su estado. A ello, las comunidades responden adoptando medidas que tratan de mantener el equilibrio ecológico más adecuado. Sus relaciones causa-efecto confluyen por medio de indicadores basados en los aspectos de la sostenibilidad, los cuales son:

- Indicadores de presión. "Describen la presión ejercida por las actividades humanas sobre el ambiente y los recursos naturales; están relacionados con los patrones de producción y de consumo". 
- Indicadores de estado. "Diseñados para mostrar imágenes (estado) de la situación que guardan el medio ambiente y los recursos naturales en el tiempo".

- Indicadores de respuesta. "Vinculados con acciones y reacciones colectivas que lleva a cabo la sociedad para mitigar, adaptar y revertir los efectos negativos de las actividades humanas sobre el medio ambiente" (Ibáñez, 2012: 106).

Su ventaja principal es su sencilla construcción basada en inventarios de información amplia y diferenciada (Ibáñez, 2012: 106). Sin embargo, la principal dificultad radica en los altos costos de monitoreo y en la poca disponibilidad de información en escalas específicas; por tanto, no generan datos precisos de la sostenibilidad global (Ivars et al., 2001: 52).

3.b.Basadosen el modelo fuerzas motoras-presión-estadorespuesta (FPEIR): este es un modelo mejorado del PER, el cual incorpora las "fuerzas motrices" — causa - de la presión sobre el medio físico (Rivas y Magadán, 2007: 52; Ibáñez, 2012: 106). Sus ventajas radican en que permite analizar distintos aspectos y dimensiones del desarrollo humano sostenible, mientras que su única desventaja radica en su operatividad en la praxis, ya que requiere de grandes cantidades de datos muy heterogéneos (Ibáñez, 2012: 107).

3.c. Según el modelo para el análisis de la sostenibilidad, basado en el barómetro de la sostenibilidad: este modelo establece que el alcance de objetivos hacia el desarrollo sostenible puede medirse a través del nivel de bienestar de la sociedad y el ambiente, por lo que propone cuarenta indicadores - principalmente PER - en los componentes social y ecológico (Ibáñez, 2012: 108). Su ventaja es que muestra mayor tendencia hacia los principios de equidad e igualdad social y ecosistémica, así como hacia sus interrelaciones. Sus desventajas se observan en la praxis, donde el mayor problema radica en la falta de información, por lo que no se puede agregar el mismo número de indicadores para cada ámbito de la sostenibilidad (Ibáñez, 2012: 108).

4. Según el enfoque en el que estén basados: 4.a. Monetarios: son indicadores de valorización monetaria sobre la amortización de los recursos y servicios ambientales y su impacto en el nivel de vida de la población. Entre sus ventajas destaca la interrelación de variables económicas con factores sociales, así como variables de captación y consumo de recursos naturales, su agotamiento y su capacidad de resiliencia. Entre los inconvenientes que enfrenta se encuentra su dificultad para implementarse a escalas territoriales pequeñas y la asignación monetaria subjetiva a los daños irreversibles al ambiente, partiendo del principio de que los bienes y servicios ambientales son intangibles y, por tanto, incuantificables monetariamente (Ibáñez, 2012: 109).

4.b. Biofísicos: miden los insumos empleados en productos y servicios de la economía relacionados con su vida útil, estableciendo niveles de consumo e impacto ambiental mediante información científica y metodológica. Entre sus ventajas destaca la medición y evaluación de impactos ambientales de origen antropocéntrico y la disponibilidad de recursos naturales para las presentes y futuras generaciones. Su principal desventaja radica en su aplicabilidad didáctica y conceptual, y en la práctica generalmente la información requerida para sus estudios no está disponible (Ibáñez, 2012: 109).

La categorización y ponderación de indicadores se basa en consensos, propuestas de expertos, y en opiniones recabadas en foros participativos o retomadas de la literatura (Ibáñez, 2012: 110). Se puede determinar una escala que va de 0 a 1 , lo cual facilita su manejo e interpretación. Cuando se reúne información de un sistema de indicadores, es factible resumirla en un promedio o índice, el cual denotará según una escala el grado de alcance hacia el desarrollo sostenible. De acuerdo con el método de la Unión Internacional para la Conservación de la Naturaleza (UICN, 2001), obtener un resultado promedio entre 0.8 y 1.0 expresa un nivel deseable. No obstante, un resultado entre 0.0 y 0.2 denota una situación totalmente indeseable, así como un fuerte rezago social, combinado con una pésima calidad ambiental (Ibáñez, 2012: 112). 


\section{Indicadores turísticos de sostenibilidad}

Los indicadores de sostenibilidad turística son herramientas de medición cuantitativa y cualitativa que permiten evaluar la tendencia de un destino hacia el alcance de la sostenibilidad y sus impactos sobre el ambiente, las comunidades y los recursos económicos generados. Su implementación permite seleccionar, sintetizar y evaluar información generada durante un tiempo, así como conducir, redirigir y reorganizar objetivos y acciones que permitan el crecimiento y el desarrollo sostenible del destino ecoturístico. Además, son necesarios para conservar y proteger los recursos naturales y la identidad cultural de las comunidades locales, así como para mantener la competitividad del destino (OMT, 1996: 74). Es importante señalar que aún hace falta un planteamiento científico aceptado por unanimidad sobre los indicadores idóneos que permitan a los gestores y operadores la evaluación de la sostenibilidad (Sancho et al., 2011: 188). Por ello, se hace necesario identificar, construir o seleccionar variables adaptadas a las condiciones locales como indicadores de valor intrínseco para su monitoreo. Dichas variables serán útiles en la supervisión y control de planes, estrategias y actuaciones turísticas (Rivas y Magadán, 2007: 9). Debemos señalar que, de acuerdo con la OMT (1996: 74), un sistema de indicadores no es un fin en sí mismo, ya que el resultado de su análisis muestra tendencias de cambio, y posibles señales de alerta y preocupación en los atributos de la sostenibilidad. Su evaluación final permite establecer estándares comparativos con normatividades u otros destinos con características similares de intensidad en uso o contaminación para realizar un intercambio de información y experiencias (OMT, 1996: 74).

\section{Características de los indicadores de sostenibilidad turística}

Es necesario que los indicadores de sostenibilidad en el turismo cumplan con características indispensables para su utilización como las siguientes: claridad, rugosidad, pertinencia, factibilidad, credibilidad, comparabilidad, representatividad, sensibilidad, valor predictivo, integrabilidad y aplicabilidad (OMT, 1996: 74). La ausencia de este proceso de análisis en la selección de indicadores puede conducir a errores de redundancia de información, lo que pondría en riesgo su validez.

\section{Clasificación de los indicadores de sostenibilidad turística}

1. Indicadores básicos: son indicadores que permiten obtener elementos clave de monitoreo en los cambios existentes en la relación hombre-naturaleza. Su desafío radica en identificar aquellos que proporcionen la información requerida. Son prácticos y básicos y en la mayoría de los sitios no debería representar mayor problema la obtención de la información que requieren. A estos indicadores se suman los indicadores complementarios.

2. Indicadores complementarios (destinos específicos): son indicadores diseñados para completar los básicos. Permiten la identificación de variables o atributos de cambios únicos para los diferentes tipos de destinos. Según la Organización Mundial del Turismo se clasifican en dos:

- Indicadores de ecosistemas específicos: se dividen en ocho grupos diferentes de acuerdo con las categorías de los ecosistemas. Entre ellos se encuentra la clasificación de sitios únicos ecológicos, los cuales frecuentemente son destinos ecoturisticos (OMT, 1996).

- Indicadores de gestión de sitios específicos: diseñados por los administradores de los destinos para su aplicación en sitios específicos, los cuales a su vez se subdividen en dos clases (OMT, 1996: 74):

* En las categorías contempladas se encuentran los ecosistemas de zonas costeras y de montaña, parques de vida silvestre, ecosistemas urbanos, patrimonios culturales, comunidades tradicionales e islas pequeñas de protección.

* Indicadores de manejo en sitios específicos: su identificación e implementación permite medir los atributos particulares de un destino 
turístico específico y, por tanto, tomar decisiones que promuevan la gestión sostenible de actividades que tengan impacto sobre estos atributos.

\section{Propuestas internacionales en la medición de la sostenibilidad ecoturística}

La actividad ecoturística presenta desafíos complejos en materia de sostenibilidad. Sus tendencias globalizadas hacia un turismo sostenible hacen necesario romper el paradigma tradicional, y se demandan metodologías básicasy específicasque permitan medir su acercamiento al modelo utópico en los destinos turísticos. En la actualidad existen organismos internacionales encargados de establecer lineamientos para el desarrollo de herramientas de medición de la sostenibilidad turística y estándares de certificación de destinos y desarrollo de espacios turísticos sostenibles (Ibáñez y Ángeles, 2012: 54). Destacan la Organización para la Cooperación y el Desarrollo Económico (OCDE), la Organización Mundial del Turismo (OMT) y la Asociación de Estados del Caribe (AEC). Esta última presentó en el año 2004 el primer Manual sobre indicadores de sustentabilidad para sus países miembros. Su metodología plantea el uso de índices de sostenibilidad categorizando los destinos turísticos posteriormente a su aplicación como: "destino turístico comprometido", con grado mínimo, medio y superior de sustentabilidad. Sin embargo, hasta la fecha los resultados de su evaluación en más de treinta países del Caribe no han sido publicados (Ibáñez y Ángeles, 2012: 55). A pesar del avance se pueden analizar vacíos, como la limitación del componente ambiental para la evaluación de la sostenibilidad, así como la relación e influencia de un índice sobre otro (Ibáñez y Ángeles, 2012: 55). La OMT, por su parte, propone principios básicos para el diseño y la selección de indicadores turísticos, y hace referencia a la importancia de su adaptación a estudios de caso (OMT, 2005: 273). Básicamente las propuestas actuales de índices de sostenibilidad a nivel mundial se fundamentan en lo establecido por la OMT y han sido adaptados por las instituciones del sector en cada país de acuerdo con sus propios paradigmas. En el caso de México se empleó la metodología establecida por la institución federal correspondiente que, a pesar de ser avalada por la OMT, ha generado poca información.

\section{Normatividad mexicana y su relación con los indicadores de sostenibilidad ecoturística}

En México, la norma NMX-AA-133-SCFI-2013 (2014) establece los requisitos y especificaciones de sustentabilidad del ecoturismo para la certificación de la actividad en su aproximación al desempeño sostenible de los destinos. Es decir, se trata de lineamientos dirigidos a las comunidades anfitrionas prestadoras de servicios en el país, que tienen como finalidad ser reconocidas en su compromiso con la sostenibilidad y la satisfacción de los visitantes con base en el cumplimiento de los estándares propuestos por la OMT.

La norma posee un listado de requisitos generales tanto para instalaciones, como para actividades ecoturísticas. Además, incluye un apéndice normativo sobre su metodología de evaluación en el que se numera cada requisito con su medio de verificación y los criterios de valoración y, finalmente, conforme al cumplimiento y entrega de los productos finales se obtiene una ponderación.

Aunque la norma es una guía de requisitos tangibles en documentaciones y productos verificables por las certificadoras, lo cierto es que tienen un amplio potencial para convertirse en indicadores básicos de sostenibilidad. Ello puede ser posible si se selecciona cada uno con base en las características de un índice, como las siguientes: factibilidad, claridad, rugosidad, pertinencia, credibilidad, comparabilidad y valor predictivo (OMT, 1996: 74).

Dentro de la lista de requisitos sobresale, debido a que cumple con las características de un indicador, aquel que se refiere al índice de capacidad de carga. Desafortunadamente, al tratarse de uníndice compuesto (OMT, 1995), conformado por al menos seis índices básicos con variables de respuesta cuantitativas y cualitativas, se dificulta su medición, lo que demanda la remisión a una metodología que permita cuantificar 
o establecer valoraciones cruzadas y objetivas para obtener una ponderación final. Subsecuentemente, requiere del establecimiento de propuestas referenciales o criterios básicos de comparabilidad para que investigadores o certificadores la acepten o rechacen, ya que se trata de un índice importante por la amplia gama de indicadores básicos que lo conforman. Vale la pena resaltar la importancia de la norma como un motivante para que las comunidades anfitrionas construyan su propio sistema de índices a fin de medir la actividad ecoturística a través del análisis de sus procesos de planificación y de la identificación de sus necesidades socioeconómicas y culturales, así como de los lineamientos de protección de los recursos ambientales. Sin embargo, una de sus mayores debilidades radica en la omisión de propuestas o parámetros de referencia para su construcción dentro de los destinos.

Consideramos que, si bien la norma NMX-AA133-SCFI-2013 es una lista de requisitos que, una vez cumplidos, permiten a un destino constituirse en apto para un proceso de evaluación, no es un referente para la medición de la sostenibilidad ecoturística. No obstante, podría convertirse en una excelente metodología si en ella se enfatizaran los lineamientos básicos para la construcción, medición y monitoreo de índices en los destinos de acuerdo con las características propias de cada uno.

\section{Caracterización del turismo en México y Chiapas: iniciativa y praxis en el uso de indicadores de sostenibilidad turística}

En México el turismo representa una fuente de divisas y empleos que contribuye con el 8\% del PIB nacional $y$, por ende, se trata de un sector que ha cobrado gran relevancia en la economía del país (Flores, 2012: 13). Sin embargo, este sector también requiere de medidas para mantener su estado competitivo a nivel internacional, donde las variables ambientales juegan un rol crucial. Por ello, es importante establecer y ejecutar metodologías de medición de impactos que permitan estimar su aproximación al paradigma de la sostenibilidad. Aunque esta preocupación responde a la tendencia globalizada de los países a establecer una economía verde, lo cierto es que, de llevarse a cabo de forma consciente, promete la conservación de los recursos naturales y la competitividad de los destinos en el tiempo.

Como resultado de las demandas internacionales del turismo en materia de sostenibilidad, relacionadas con la creación de políticas orientadas al cuidado del ambiente, la Secretaría de Turismo (SECTUR) en México impulsó el Programa Agenda 21 en más de 72 municipios turísticos. El Modelo de Indicadores de Sustentabilidad para el Turismo diseñado por esta misma institución tuvo como objetivos medir y monitorear las condiciones de cada destino turístico. Básicamente, incluye un programa para la formulación de indicadores dependiendo del tipo de destino, su aval a través de una red de expertos y actores locales, un análisis de viabilidad considerando escalas territoriales, límites y criterios de ponderación. Sin embargo, de acuerdo con Ibáñez y Ángeles (2012: 59), estas herramientas no consideran una relación clara entre los mismos ni el impacto que se genera entre indicadores de distinta naturaleza. Cabe señalar que desde su aplicación en 2008 hasta la fecha aún hace falta bibliografía en la que se divulguen sus resultados.

En el contexto regional, la influencia y el apoyo de organizaciones internacionales en la investigación sobre el sector turístico han propiciado el impulso de planes de estrategia y competitividad para conglomerados turísticos en Chiapas. En ellos se realiza un análisis general del entorno en algunos destinos de un área específica, así como de su potencial natural y cultural (Plan de Estrategia y Competitividad Turística, 2009: 24). Sin embargo, sus áreas de estudio se limitan a sitios de turismo cultural, de reuniones y natural. En los sitios de turismo natural establecen objetivos y acciones con tendencia a la sostenibilidad y a la protección del ambiente y la biodiversidad. Por ejemplo, la propuesta de desarrollo para el segmento del ecoturismo en Chiapas, con base en el análisis realizado, se fundamenta en el incremento de infraestructura adecuada al entorno para alojamiento, campamentos, 
viviendas locales o cabañas, gastronomía y actividades relacionadas con el entorno natural y cultural. Además se proponen actividades complementarias, como labores de agricultura y ganadería ecológicas, técnicas artesanales, sistemas ecológicos de interés y acciones de conservación y recuperación de espacios (Plan de Estrategia y Competitividad Turística, 2009: 142). No obstante, en ningún punto de la propuesta se hace referencia a un análisis del entorno mediante un sistema de medición y monitoreo de los atributos ambientales y socioculturales actuales y futuros de los destinos, ni tampoco a las necesidades de las comunidades locales y su relación con la actividad. Relega también aspectos importantes, como el respeto a la autenticidad cultural de las comunidades anfitrionas, el aseguramiento de actividades rentables en el tiempo y el uso óptimo de los recursos ambientales como ejes rectores de la sostenibilidad ecoturística (OMT, 2005: 268).

Aunque los indicadores de sostenibilidad turística no han sido diseñados, seleccionados, evaluados y monitoreados en su concepción másintrínsecaen Chiapas, existe un estudio sobre un caso cercano. La investigación realizada por Alarcón (2010)enel Centro Ecoturístico Sima de las Cotorras en Ocozocoautla de Espinosa, Chiapas, es la primera aproximación a la utilización de indicadores sociales a esa escala, de manera que, a través de diversas herramientas, la autora obtuvo información cualitativa y cuantitativa, y analizólasimplicaciones y contradicciones del centro mediante un proceso participativo para la toma de decisiones con diferentes actores sociales. Los resultados a los que llegó lainvestigación evidenciaron que existen problemas constatables de desorganización social y, por tanto, de comunicación y de desconocimiento de los lineamientos del ecoturismo, además de una imposición de instituciones de gobierno sobre la cooperativa. En el contexto del desarrollo turístico estatal, así como por las condiciones socioeconómicasimperantesen Chiapas, este hallazgo resulta más que esclarecedor.

\section{Indicadores de sostenibilidad y políticas turísticas}

En el año 2000, a nivel nacional la metodología del Instituto Nacional de Estadística, Geografía e
Informática (INEGI) auspiciada por la Comisión de Desarrollo Sustentable (CDS) de Naciones Unidas fue dada a conocer con 113 indicadores para medir la sustentabilidad en México (Ibáñez y Ángeles, 2012: 51). Este proceso de diseño y construcción de indicadores de sostenibilidad se llevó a cabo con diferentes organismos institucionales nacionales y extranjeros, tales como el INEGI, el hoy Instituto Nacional de Ecología y Cambio Climático (INECC), la Secretaría del Medio Ambientey Recursos Naturales (SEMARNAT), el Banco Mundial (BM), la Organización de las Naciones Unidas para la Educación, Ciencia y la Cultura (UNESCO), la Organización de las Naciones Unidas para la Agricultura y Alimentación (FAO) y el Programa de las Naciones Unidas para el Medio Ambiente (PNUMA), entre otros.

En este punto vale la pena mencionar que resulta altamente complejo romper con paradigmas arraigados. La concepción tradicional del turismo suele remitirse a la planeación gubernamental y a la aplicación de políticas superficiales que redundan en el clásico discurso político.

El inconveniente para México en materia de indicadores radica en los acuerdos internacionales firmados en relación con el cambio climático y con el alcance de los objetivos de desarrollo del milenio, además de que las acciones locales para alcanzar los retos nacionales requieren de articulación interinstitucional. Como ya se mencionó en el apartado anterior, en el caso del sector turístico su medición corrió a cargo de la SECTUR con la conformación del Programa Agenda 2l. Es relevante mencionar que en el proceso de diseño y construcción de los indicadores de sostenibilidad turística, además de la validación de la OMT, existe nula información bibliográfica que confirme si fueron construidos con la participación interinstitucional; es decir, si en el consenso se contó con aportaciones de representantes del Instituto Nacional de Derechos Humanos (INDH), de la Secretaria de Desarrollo Social (SEDESOL), del Instituto Nacional de las Mujeres (INMUJERES) y de la Comisión Nacional para el Desarrollo de los Pueblos Indígenas (CDI) o, más importante aún, de 
representantes del sector turístico que, para agregar complejidad, debieran ser de orígenes tanto urbanos como rurales, tanto de esfuerzos intensivos como comunitarios o de baja escala, tanto usuarios de ecosistemas áridos o semiáridos, como de las selvas tropicales del país.

En la actualidad las políticas públicas turísticas se enfocan en el llamado de la OMT para reducir las emisiones de gases de efecto invernadero, ya que el sector turístico contribuye con el cinco por ciento del total de emisiones que provocan el cambio climático (Geiger e Ibáñez, 2012: 35). Si bien se aplican normatividades relacionadas con la gestión de calidad en el cumplimiento de indicadores básicos para las demandas internacionales sobre cambio climático, lo cierto es que dichos datos son insuficientes para categorizar un destino como sostenible. Es decir, hace falta considerar aspectos diferentes a los técnicos.

La falta de información nacional divulgada sobre los resultados de la evaluación de los destinos con indicadores de sostenibilidad turística y su implicación en el desarrollo de políticas públicas deja en entredicho las áreas de oportunidad que aún puedan existir para un mejoramiento de la praxis. Es indispensable no sólo la ejecución, sino también la evaluación de las propuestas de indicadores existentes.

El turismo es un complejo sinérgico de relaciones y actividades sociales, económicas, ambientales, culturales, administrativas y políticas con implicaciones sobre la dinámica social y el modus vivendi de las comunidades. Su estado refleja en cierta medida la instrumentación e intervención de políticas impuestas por una economía globalizada (Mathias y Salama, 1986: 29-30). Por ello, es importante analizar la complejidad de las políticas turísticas y referirse a cuestionamientos organizacionales e institucionales en los que se asume que las decisiones surgen a partir de un proceso democrático para alcanzar metas (Castillo et al., 2008: 94).

La relación entre ecoturismo y políticas va más allá de la aplicación de programas de promoción, de satisfacción de los visitantes y de inversión en infraestructura; se requiere de esfuerzos por invertir en la preparación de recursos profesionales en el diagnóstico, planeación, diseño, evaluación y monitoreo de los destinos turísticos para garantizar su sostenibilidad y competitividad en el tiempo, así como su impacto positivo en la calidad de vida comunitaria.

La selección, diseño, construcción, medición y evaluación de indicadores de sostenibilidad turística requiere de un análisis metodológico básico, complementario y particular si se desea contar con estadísticas regionalizadas e institucionales. Estas herramientas serán útiles para monitorear un determinado proceso de evaluación de sostenibilidad pero, asimismo, lo serán para el tipo de política turística y sus esquemas de desarrollo a nivel global y local.

Si el proceso de evaluación es local, la metodología debe consistir en tres etapas:

1) Investigación y organización. Esta etapa incluye el proceso de recopilación de datos del destino y la realización de una lista de preindicadores básicos y complementarios - exclusivos para el sitio-; debe verificarse su viabilidad, la disponibilidad de información, y que además cumplan con las características de un buen indicador (OMT, 2005: 268). Además, se lleva a cabo la identificación de temas prioritarios y de los atributos del sitio con base en aspectos socioambientales. Los atributos son el conjunto de características que proporcionan la base contra la cual se miden los indicadores a través del tiempo; es decir, los cambios que se susciten en los atributos medidos por los indicadores proveen información directa sobre la sostenibilidad de las actividades relacionadas con el turismo. Para ello se requiere de herramientas cualitativas, como entrevistas semiestructuradas tanto a visitantes, como a personas locales y miembros de la cooperativa o administración del destino turístico.

2) Selección y adaptación de indicadores. En esta etapa se obtiene la lista final de indicadores básicos y complementarios con base en la información obtenida y disponible de la etapa anterior a partir de la lista de indicadores preseleccionados. Además, se realiza la adaptación de algunos de ellos mediante el consenso entre expertos en temas como los siguientes: ambiente, sociedad, turismo, sostenibilidad y economía. 
3) Aplicación y medición de indicadores.Una vez obtenida la información de cada indicador a través de las herramientas y métodos ya descritos en etapas anteriores, se procederá a su medición. En el caso de valores establecidos para los atributos sociales, estos deben ser consensuados - procedimiento complementario de las mediciones científicas-técnicas involucradasLa evaluación final consiste en establecer un valor ideal para cada indicador, y se debe realizar el cálculo de la proporción que corresponda a cada valor obtenido de la medición del indicador con respecto al ideal. Es decir, que cuanto más se acerque la medición del indicador a su valor ideal, se estará más próximo a una óptima operatividad para ese indicador. El resultado se debe comparar con el del estado óptimo para el mismo destino, en caso de no existir otro con características o atributos similares y que además haya evaluado su sostenibilidad. Con el resultado de su interpretación, se deben tomar medidas sobre el encauzamiento de actividades, prácticas y políticas normativas que deban implementarse para potenciar el desarrollo de la sostenibilidad y competitividad del destino.

En cambio, si la evaluación de los indicadores de sostenibilidad turística es regional, entonces, además de la existencia de un padrón de destinos evaluados, debe haber una categorización de los destinos con respecto a atributos semejantes o bien con respecto al tipo de ecosistema que comparten entre ellos para ser comparados. Por ejemplo, es insuficiente comparar la playa de Acapulco con respecto a otra en la península de Yucatán que cuente con una zona arqueológica. Otro caso similar sucedería con un destino situado en un bosque de niebla y otro en una selva baja caducifolia; aunque ambos contaran con un atributo social común, la biodiversidad marcaría la diferencia. Lo mismo podría suceder en comunidades con la misma lengua nativa, pero en diferente ubicación geográfica y con distintos atributos ambientales.

\section{Conclusiones}

Es evidente la importancia de la falta de un sistema de medición de sostenibilidad turística estandarizado en lo general y con especificidades en lo particular. Por un lado, se carece de información respecto de la cercanía de los centros ecoturísticos, o bien del manejo de dichos centros, con el paradigma de la sostenibilidad. Actualmente, no existe en México un documento derivado de un estudio minucioso que aporte información a este respecto sobre la mayoría de los destinos turísticos en el país.

Por otro lado, esta carencia también hace obvia la limitada capacidad de los actores sociales involucrados en la actividad ecoturística por mejorar los procesos existentes, particularmente aquellas mejoras tendientes a beneficiar a los gestores comunitarios sin menoscabo de los recursos naturales del destino. Estos vacíos cobran relevancia en el escenario actual del turismo en México, en el que el país, de acuerdo con la OMT (2014), se ubica por arriba del resto de Latinoamérica en el número de llegada de turistas.

Las propuestas para llenar este vacío de información son diversas, y es necesario fomentar la discusión y el análisis en torno a las fortalezas y debilidades de cada una de ellas. En ese sentido, proponemos que la construcción de dicho sistema de medición se fundamente en paradigmas de planeación turística distintos, que prioricen el ambiente y sus recursos por encima del componente económico, y que en todos los casos considere la intervención consensuada de todos los actores sociales.

Finalmente,esimportanteseñalarquelaconstrucción e implementación de un sistema de indicadores de sostenibilidad turística puede convertirse en un medio para el diálogo entre diseñadores de políticas, tomadores de decisiones y gestores locales ante las necesidades, prioridades y demandas ambientales, económicas y socioculturales en cada destino ecoturístico.

\section{Referencias bibliográficas}

Alarcón, Perla (2010). Implicaciones y contradicciones del ecoturismo en la Sima de las Cotorras, Ocozocoautla de Espinoza, Chiapas. Tesis de Maestría en Ciencias en Recursos Naturales y Desarrollo Rural, El Colegio de la Frontera Sur, México. 
Angulo, Nicolás (2010). "Pobreza, medio ambiente y desarrollo sostenible". En Nómadas. Revista crítica de ciencias sociales y jurídicas, núm. 26. Madrid. Disponible en: http://www.redalyc.org/articulo. oa?id=18118916003 (consultado el ll de enero de 2015).

Blancas, Francisco, Mercedes González, Flor Guerrero y Macarena Lozano (2010). "Indicadores sintéticos de turismo sostenible: Una aplicación para los destinos turísticos de Andalucía”. En Revista Electrónica de Comunicaciones y Trabajos de ASEPUMA, núm. 11. España. Disponible en: http://www.revistarecta.com/ nll/recta_ll_09.pdf (consultado el 14 de diciembre de 2014).

Constanza, Robert (1991). "Assuring Sustainability of Ecological Economic Systems”. En Robert Constanza y Lisa Wainger (coord.), Ecological Economics: The Science and Management of Sustainability. Nueva York: Columbia University Press, pp. 331-343.

Daly, Herman (1992). "Steady-State Economics". Londres: Earth Scan.

"Declaratoria de vigencia de la norma mexicana NMXAA-133-SCFI-2013, requisitos y especificaciones de sustentabilidad del ecoturismo (cancela a la NMXAA-133-SCFI-2006)" (2014). En Diario Oficial, primera sección, lunes 7 de abril de 2014.

González, José, Carlos Montes e Ignacio Santos (2007). "Capital natural y desarrollo: por una base ecológica en el análisis de las relaciones Norte-Sur". En Papeles de Relaciones Ecosociales y Cambio Global, núm. 100. España. Disponible en: https://www.uam.es/gruposinv/ socioeco/documentos/papeles.pdf (consultado el 26 noviembre de 2014).

Guillén Romo, Arturo (2004). "Revisitando la teoría del desarrollo bajo la globalización”. En Economíaunam, núm. 1, pp. 19-42. México.

Ibáñez, Reyna (2012). "Teoría y praxis. Indicadores de sustentabilidad: utilidad y limitaciones". En El Periplo Sustentable, núm. 1l, pp. 47-66. México: UABC.

Jiménez, Luis (2000). Desarrollo sostenible. Transición hacia la coevolución global, vol. 1. España: Pirámide.

Luffiego, Máximo y José María Rabadán (2000). "La evolución del concepto de sostenibilidad y su introducciónenlaenseñanza”. EnEnseñanzadelasCiencias, núm. 18. España. Disponible en: http://www.raco.cat/ index.php/ensenanza/article/viewFile/21701/21535 (consultado el ll de enero de 2015).

Mendieta, Juan Carlos (2000). Economía ambiental. Santa Fe de Bogotá: Facultad de Economía-Universidad de los Andes.

Naredo, José Manuel (1996). "Sobre el origen, el uso y el contenido del término sostenible". En Documentación Social, núm. 102, pp. 129-142.

OMT (Organización Mundial de Turismo) (1996). "Lo que todo gestor turístico debe saber". En OMT (coord.), Guíaprácticapara el desarrolloy uso de indicadores de turismo sostenible. Madrid: OMT.

OMT (Organización Mundial de Turismo) (2005). "Indicadores de desarrollo sostenible para los destinos turísticos”. En OMT (coord.), Guía práctica. Madrid: OMT.

OMT (Organización Mundial de Turismo) (2014). Panorama OMT del turismo internacional, edición 2014. Madrid: OMT

Rivas, Jesús y Marta Magadán (2007). "Los indicadores de sostenibilidad en el turismo", En RevistadeEconomía, Sociedad, Turismo y Medio Ambiente: RESTMA, núm. 6, pp. 27-62. España.

Rostow, Walt (1960). The Stages of Economic Growth, A Non-Communist Manifiesto. Cambridge: Cambridge University Press.

Sánchez Rivero, Marcelino (2008). "Construcción de índices sintéticos de sostenibilidad turística mediante modelos factoriales: aplicación al Sistema Español de Indicadores Ambientales de Turismo". En Las administraciones públicas y empresas turísticas ante el reto de la sostenibilidad. España: Universidad Católica San Antonio de Murcia, Asociación Española de Expertos Científicos en Turismo, AECIT. Disponible en: http:// dialnet.unirioja.es/servlet/articulo?codigo=3123468 (consultado el ll de enero de 2015).

Sancho, Amparo et al. (2008). Introducción al turismo, t. 1. Madrid: OMT

SECTUR (Secretaría de Turismo) (2014). "Planeación y política turística”. México: SECTUR. Disponible en http://www.sectur.gob.mx/programas/planeacion-ypolitica-turistica/ (consultado el 4 de agosto del 2014). 
Toro, Francisco Javier (2007). "El desarrollo sostenible: un concepto de interés para la geografía”. En Cuadernos Geográficos, núm. 40, pp. 149-181.

UICN (Unión Internacional para la Conservación de la Naturaleza) (2001). "Resource Kit For Sustentabilily Assessment”. Suiza: UICN. Disponible en: http:// cmsdata.iucn.org/downloads/resource_kit_a_eng.pdf (consultado el ll de enero de 2015).

Vanegas Montes, Gloria Mercedes (2006). "Ecoturismo, instrumento de desarrollo sostenible". Proyecto para optar al título de Especialista en Gestión Ambiental, Facultad de Ingeniería de la Universidad de Antioquía, Colombia. Disponible en: http://www.researchgate.net/ publication/37368330_Ecoturismo_instrumento_de desarrollo_sostenible (consultado el 11 de enero de 2015).
Vera Rebollo, José Fernando (2001). "Planificación y gestión del desarrollo turístico sostenible: propuestas para la creación de un sistema de indicadores". En Documentos de trabajo del Instituto Universitario de Geografía, núm. 1, España.

Wolfensberger, Lilly (2005). Sustentabilidad y desarrollo. Suficiente siempre, t. l. México: Porrúa.

UNWTO (World Tourism Organization) (2009). "Plan de estrategia y competitividad turística para los clusters de Tuxtla Gutiérrez, San Cristóbal de las Casas, Palenque, Comitán de Domínguez y Chiapa de Corzo, Chiapas". México: UNWTO. Disponible en: http:// www.turismochiapas.gob.mx/sectur/media/ descargables/omt/Chiapas2015 (consultado el ll de enero de 2015). 